\title{
Toward a Process-Based Molecular Model of SiC Membranes. III. Prediction of Transport and Separation of Binary Gaseous Mixtures Based on the Atomistic Reactive Force Field
}

\author{
Saber Naserifar, ${ }^{\dagger, \S}$ Theodore T. Tsotsis, ${ }^{\dagger}$ William A. Goddard III,,$^{\S}$ and \\ Muhammad Sahimi ${ }^{\dagger}, \mathbb{\pi}$ \\ ${ }^{\dagger}$ Mork Family Department of Chemical Engineering $\& 3$ Materials Science, University of \\ Southern California, Los Angeles, California 90089-1211, \\ $\S$ Materials and Process Simulation Center, California Institute of Technology, Pasadena, \\ California 91125
}

\begin{abstract}
The atomistic model of amorphous silicon-carbide membrane that was developed in Parts I and II of this series is utilized in nonequilibrium molecular dynamics (MD) simulations to study transport and separation of equimolar gaseous mixtures $\mathrm{H}_{2} / \mathrm{CO}_{2}$ and $\mathrm{H}_{2} / \mathrm{CH}_{4}$ in the membrane at high temperatures. We simulated membranes with up to about $39 \mathrm{~nm}$ in thickness, containing up to 170,000 atoms, and up to 100 nanoseconds to obtain reliable statistics. The effect of such parameters as the temperature, the applied pressure drop across the system, and the membrane's thickness on the separation properties was studied. The trends in the dependence of the separation factor on the membrane's thickness are consistent with experiments, namely, the separation factor increases with the thickness up to an optimal value, beyond which it remains constant, or may even decrease. The dependence of the computed separation factor on the membrane's thickness is used in conjunction with the experimental values of the separation factor to estimate the thickness of actual membranes. The results are in agreement with the experimental data, demonstrating the value of MD simulations with a reactive force field for characterizing the properties of complex amorphous films, and flow and transport therein.

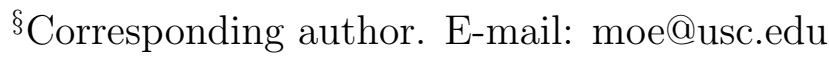

(C) 2014. This manuscript version is made available under the Elsevier user license http://www.elsevier.com/open-access/userlicense/1.0/ 
from those under bulk conditions. Second, it is of clear practical interest to understand the mechanism of transport in the pore space of nanoporous materials, since they are studied intensively for a wide variety of applications. Third, the flow and transport properties provide a probe for characterizing the morphology of the pore space of nanoporous materials.

The SiC membranes that we study contain pores with an average size of a few $\AA$. Due to such exceedingly small pore size, which is comparable with that of the gases under consideration, the main resistance to the flow and transport is controlled by its nano- and micropores. Consequently, the molecular interactions between the gases, and between the gases and the pores' walls are important and must be included. Hence, one must utilize atomistic modeling and simulation to study the transport processes that take place in the membranes' pore space $[11]$.

Thus, in the present paper we use atomistic simulations to study transport and separation of the aforementioned binary mixtures through the SiC membrane model generated in Parts I and II. Here, we apply an external pressure to the membrane to induce separation of the gases in the membrane as they are transported through the membrane, hence requiring nonequilibrium molecular dynamics (NEMD) simulations.

The rest of this paper is organized as follows. Section 2 describes the molecular models, and the procedure for the NEMD calculations. Section 3 presents preliminary results that are utilized to set the parameters of the large-scale MD simulations to be reported in Sec. 4. Then, Sec. 4 describes the approach to steady-state conditions and the implications of the results. The main results, including the effect of the various factors on the separation properties of the membrane, are presented in Section 5, where we also compare the results with the experimental data. The paper is summarized in the last section.

\section{Molecular Models and Dual Control Volume-Grand Canonical Molecular Dy- namics Simulation}

We utilized the dual control volume-grand canonical molecular dynamics (DCV-GCMD) method to study transport and separation of the binary gaseous mixtures in the SiC membranes. This method combines MD and Monte Carlo (MC) simulations [12-14], making it ideal for nonequilibrium systems under such external driving forces as a pressure or chemical potential gradient [15-20]. In this method the system under consideration is partitioned into three regions, 
as shown in Figure 1. The origin of the coordinates is taken to be the system's center. The external driving force is a pressure gradient, applied in the direction of flow and transport (the $x$ direction). The control volumes (CVs) on the left and right sides represent, respectively, the high- and low-pressure regions, which are referred to as $h$ and $\ell \mathrm{CVs}$. They are in equilibrium with the bulk condition. The middle region represents the amorphous SiC membrane, generated in Parts I and II. Its length (thickness) is $n L$ with $n$ an integer, $n=1-10$. Periodic boundary conditions were employed in the $y$ and $z$ directions. Note that such a model accounts for the pore entrance effects.

The gases and the atoms of the membrane are described by Lennard-Jones (LJ) spheres, characterized by effective LJ size and energy parameters, $\sigma$ and $\varepsilon$. The standard cut-and-shifted 6-12 LJ potential was used to model the molecule-molecule interactions:

$$
U(r)=\left\{\begin{array}{cc}
U_{L J}(r)-U_{L J}\left(r_{c}\right), & r \leq r_{c} \\
0, & r>r_{c}
\end{array}\right.
$$

where, $U_{L J}(r)$ is the standard 6-12 LJ potential,

$$
U_{L J}(r)=4 \varepsilon\left[\left(\frac{\sigma}{r}\right)^{12}-\left(\frac{\sigma}{r}\right)^{6}\right]
$$

$r$ is the distance between the interacting pair, and $r_{c}$ is the cut-off distance that, after some preliminary simulation, was set to be $9.6 \AA \simeq 2.5 \sigma_{\mathrm{CH}_{4}}$. Values of the LJ parameters are listed in Table 1. For unlike pairs of the atoms, the Lorentz-Berthelot mixing rules, $\epsilon_{i j}=\left(\epsilon_{i} \epsilon_{j}\right)^{1 / 2}$, and $\sigma_{i j}=\frac{1}{2}\left(\sigma_{i}+\sigma_{j}\right)$, were used to calculate the size and energy parameters of the pairs. All the quantities of interest were made dimensionless with the help of $\sigma$ and $\varepsilon$ of one of the fluids (denoted by superscript asterisk in the figures). The Verlet velocity algorithm was used to integrate the equations of motion. The $\mathrm{Si}$ and $\mathrm{C}$ atoms in the membrane itself remained, of course, frozen in their place.

Since the main purpose of using the DCV-GCMD technique is to study the effect of an external pressure gradient on the separation properties of the membrane, the contribution of temperature gradient to the transport is eliminated by holding the temperature constant in the CVs, as well as within the membrane. This was achieved by rescaling the velocity components independently in all the three directions during the entire MD simulation.

The density of each gaseous component in the two CVs must be held at some fixed values in equilibrium with the two bulk phases, and are directly related to the required chemical 
potentials and pressures in the corresponding CVs. To obtain constant values of the densities in the two CVs, sufficient number of the grand canonical (GC) MC insertions and deletions of the molecules were carried out in the CVs. The probabilities of inserting and deleting a particle of component $i$ are, respectively, given by

$$
p_{i}^{+}=\min \left\{\frac{Z_{i} V_{c}}{N_{i}+1} \exp \left(-\Delta E / k_{B} T\right), 1\right\}
$$

and

$$
p_{i}^{-}=\min \left\{\frac{N_{i}}{Z_{i} V_{c}} \exp \left(-\Delta E / k_{B} T\right), 1\right\},
$$

where $\Delta E$ is the potential energy change resulting from inserting or deleting a particle, $V_{c}$ the volume of the CV, $N_{i}$ the number of atoms of component $i$ in each CV, $k_{B}$ the Boltzmann's constant, and $Z_{i}$ is the absolute activity at temperature $T$. Values of $Z_{i}$ are calculated by

$$
Z_{i}=\frac{\exp \left(\mu_{i} / k_{B} T\right)}{\Lambda_{i}^{3}},
$$

where $\Lambda_{i}$ and $\mu_{i}$ are, respectively, the de Broglie wavelength and chemical potential of component $i$. The pressures were converted to equivalent chemical potentials using the Soave equation of state [21], according to which the pressure $P$ is related to the temperature and volume $v$ by

$$
P=\frac{R T}{v-b}-\frac{a}{v(v+b)},
$$

where $R$ is the gas constant, and $a$ and $b$ are, respectively, the energy and volume parameters of the equation. The standard mixing rules for a mixture, namely, $a=\sum_{i} \sum_{j} y_{i} y_{j} a_{i j}$ and $b=\sum_{i} y_{i} b_{i}$, were used, with $a_{i j}=\left(1-\delta_{i j}\right) \sqrt{a_{i} a_{j}}$, where $\delta_{i j}$ is the mixing parameter that we took to be zero. (Other values of $\delta_{i j}$ have been reported in the literature, though they are all very small as well.) The Maxwell-Boltzmann distribution was used to assign a thermal velocity to every newly inserted particle in a $\mathrm{CV}$ at the given $T$.

It is crucial in the DCV-GCMD simulations to select an appropriate value for the ratio $\mathcal{R}$, which is the number of GCMC insertions and deletions in each CV relative to the number of the MD steps between successive GCMC steps. This ratio must be chosen such that the correct density and pressure in the CVs are created and maintained during simulations. The optimal value of $\mathcal{R}$ in our simulations was determined to be 10:1. By optimal we mean the smallest value of $\mathcal{R}$ that yields the correct results with computational efficiency. It is important to carry out an enough number of insertions and deletion during GCMC in order to generate and 
maintain the appropriate densities in the CVs. A large $\mathcal{R}$ would require, however, expensive long calculations, while a small $\mathcal{R}$ would not generate the right conditions in the two CVs.

During the MD simulations the molecules crossing the outer boundaries of the CVs in $x$ direction were removed. The number of such particles was, however, very small compare to the total number of molecules that were inserted or removed during the GCMC simulations. In addition, for each component and in the membrane region a nonzero streaming velocity the ratio of the flux to the concentration of each component - was allowed, consistent with the presence of bulk pressure/chemical potential gradients along the flow direction. The absence of nonzero streaming velocity results in severely underestimated fluxes in the transport region. The actual streaming velocities of the molecules in the membrane region were calculated by the MD simulations [15-20].

The DCV-GCMD simulations are computationally expensive. Thus, the computation time was reduced by discretizing the simulation box into $n_{x} \times n_{y} \times n_{z}$ grid points, with the distance between two consecutive grid points equal to $0.43 \AA$. The interactions between the gases and the $\mathrm{Si}$ and $\mathrm{C}$ atoms were calculated at each of the grid points, and 3D piecewise cubic Hermite interpolation [22] was used for interpolating the results between the grid points. The results were then saved and used in the simulations. We used a (dimensionless) time step, $\Delta t^{*}=10^{-3}$, equivalent to $\Delta t \simeq 1.37 \times 10^{-3}$ ps. The fluxes were calculated at three locations along the membrane - at the entrance to the membrane, and in its middle and at the exit - and were then averaged. The flow and transport process was considered to have reached the steady state when the fluxes computed at various $y z$ planes were within $5 \%$ from the averaged value. The equations of motion were integrated over a large number of steps, up to $10^{8}$ steps, within which the steady state was achieved. The number of Si plus $\mathrm{C}$ atoms in the simulations varied from about 640 to $1.7 \times 10^{5}$. Such long simulations with a large number of atoms made it possible to identify a computational strategy that yielded results to be compared with the experimental data. They also made it feasible to study the behavior and flow and transport properties of the membrane under the conditions that are not easily accessible experimentally.

We computed such quantities of interest as the density profiles, the gas fluxes, and the separation factor of the membrane. The density profile $\rho_{i}^{z}(x)$ of gas $i$ was computed by dividing the simulation box in the $x$ direction into a resolved grid, and averaging the number of particles of component $i$ over the distance between two neighboring grid points for each MD step. The 


\section{Preliminary Simulation}

The most important contributing factors to the separation properties of a membrane are its thickness, as well as the magnitude of the applied pressure gradient and the temperature at which the separation operation is carried out. Thus, we varied the three factors to study their effect. They included simulations under external pressure drops that varied from 2 atm to $10 \mathrm{~atm}$ with increments of $2 \mathrm{~atm}$, and those ranging from $10 \mathrm{~atm}$ to $100 \mathrm{~atm}$ with increments of $10 \mathrm{~atm}$. In all simulations the pressure drop across the membrane was fixed by setting the upstream pressure in the $h$-CV equal to the target pressure drop, and the downstream pressure to zero. This provides a better comparison with the experiment in which the gases exit to a large vacuum at zero pressure.

Many simulations were carried out at $25^{\circ} \mathrm{C}$, as well as other temperatures, ranging from $100^{\circ} \mathrm{C}$ to $400^{\circ} \mathrm{C}$ with increments of $100^{\circ} \mathrm{C}$. The membrane's thickness was varied from about 
$21.4 \AA$ to $128.4 \AA$, with increments of $21.4 \AA$, and from $128.4 \AA$ to $1284.2 \AA$ with increments of $128.4 \AA$. The membrane linear size in the $y z$ plane perpendicular to the direction of the applied pressure gradient was also increased from $21.4 \AA$ with increments of $21.4 \AA$ to $64.2 \AA$ (see below).

As we describe shortly, preliminary simulations indicated that the size of the original molecular model of the SiC membranes generated in Parts I and II was not adequate enough for quantitative simulations. Thus, we increased the size of the original molecular model of the $\mathrm{SiC}$ membrane to improve the statistics of the simulations, allow a reasonable number of gas molecules to flow through the system, and to dynamically reach the steady-state condition. Thus, a membrane layer with increasing sizes was constructed by replicating the original model along the three directions until a reasonable size in each direction and for each simulation was achieved. After extensive preliminary simulations, we determined that a reasonable and computationally-affordable cross section of the membrane in the $y z$ planes (perpendicular to the flow and transport in $x$ direction) was of size $64.2 \times 64.2 \AA^{2}$. Then, membranes with various thicknesses along the $x$ direction were constructed using the identified cross section. We emphasize that a goal of the study is to understand how the separation properties of the membrane vary as a function of its thickness.

\section{Steady-State Condition}

As the first step, one must establish that simulations are carried out correctly in the sense that, the system has reached the steady-state (SS) condition, with constant values of the pressure, temperature, density, and fluxes in the two CVs and, consequently, a constant separation factor. Reaching the SS condition by the GCMD method for the present application proved to be very difficult. Experimentally, the properties are measured for membranes with cross sections and thicknesses of several $\mathrm{cm}^{2}$ and $\mu \mathrm{m}$, respectively, representing a molecularly-large system with billions of atoms. Moreover, the experiments must be carried out for at least several minutes before collecting any data, in order to ensure that the system has reached the SS condition. Therefore, it should be clear that it would be computationally impossible to simulate a system with the size and time scale that are comparable with the experimental conditions, as such simulations are well beyond the capabilities of today's supercomputers. In addition, one more point related to the nature of GCMD simulations requires care. During 
GCMD simulations, the MC moves for inserting and deleting gas molecules in the CVs occur every several MD steps, in order to set the required pressures and densities in the two CVs. Since this is done by random insertion and deletion of the gas particles in the two CVs, it causes the fluid flow and transport to experience disturbances after every several time steps, as a result of which the simulations slow down while trying to reach the SS condition.

One solution to this problem is to have sufficiently large CVs and to apply higher external pressures than in the experiment. This provides a sufficiently large number of gas molecules in the CVs that, in turn, provides better statistics after averaging over specified period of times. More importantly, the disturbance caused by the MC moves is minimized. On the other hand, enlarging the system increases the cost of the computations. In addition, to reach the SS condition, the simulations in most cases had to be carried out for more than $100 \mathrm{~ns}$, with the averages taken every one million steps.

Figures 2 and 3 present the dependence of the separation factors on the time for binary mixtures of $\mathrm{H}_{2} / \mathrm{CO}_{2}$ and $\mathrm{H}_{2} / \mathrm{CH}_{4}$, calculated for several pressure drops. As shown, in all the cases, after about 30 million time steps, the separation factors attain constant values, indicating that the SS condition has been reached.

As already pointed out, it is essential to maintain the density of each component in the two CVs at some fixed values, which are in equilibrium with two bulk phases, each at the fixed pressure and gas concentrations corresponding to the individual CVs. The densities, or the corresponding chemical potentials of each component in the CVs, were held constant by carrying out a sufficient number of the GCMC insertions and deletions of the molecules. Figure 4 present the dimensionless density profiles $\rho_{i}^{*}(x)$ for the binary mixture $\mathrm{H}_{2} / \mathrm{CO}_{2}$ at two temperatures and a pressure drop, $\Delta P=20 \mathrm{~atm}$, while Figure 5 presents the profiles for two different pressure drops, $\Delta P=60 \mathrm{~atm}$ and $100 \mathrm{~atm}$, at a same temperature. All the qualitative trends of the density profiles are the same at the two temperatures shown, except that the densities are lower at the higher $T$, which is expected. In addition, the density profiles are essentially flat in the $h$ (left) $\mathrm{CV}$ in the region $-25<X^{*}<-8.33$, and are negligible in the (right) downstream $\ell \mathrm{CV}$ in the region $8.33<X^{*}<25$ as the pressure there is zero. Although there are small fluctuations in the densities there, the numerical values of their averages match those obtained with the GCMC method at the same conditions, indicating that the chemical potentials in the two CVs are properly maintained during the DCV-GCMD simulations. 
of multiple realizations of the model membrane. In other words, once the force field, the molecular model of the polymer precursor, and the system size are specified, the MD simulation always produces the same membrane structure. The only stochastic element in the simulation is the original structure of the polymer. As described in Part II, we initially generated a molecular model of the polymer and then used MD simulation and energy minimization in order to arrive at the equilibrium structure of the polymer. To further check that the true global minimum energy of the polymer had been reached, we also used simulated annealing [23], which is a powerful computational algorithm for determining the global minimum energy state of a disordered system. Of course, similar to any other computer simulations, there are always numerical round-off errors. In addition, one must set a criterion for the system to achieve its global minimum energy state such as, for example, setting the relative change in the total energy of the system to be smaller than a small number $\epsilon$, e.g. $10^{-3}$ over a number of time steps. But, once $\epsilon$, the force field used in the MD simulation and the temperature of the system are specified, then, regardless of the initial structure of the polymer, its global minimum energy state is the same, because the true global minimum energy of the material is unique. In fact, as reported in Part I, and will be reported in a separate paper [24] in the near future, the calculated physical properties of the $\mathrm{SiC}$ material that we have generated, ranging from its density, to x-ray diffraction pattern, and radial distribution function, are all in excellent agreement with experimental data, hence indicating that the correct molecular structure of the material has been generated by our MD computations.

\section{Effect of Various Factors on Membrane Properties}

We next present the effect of various factors on the properties of the membranes. Two of the most important properties are the separation factors and the gases' fluxes.

\subsection{Temperature}

The permeability $K$ of a species is typically represented as the product of the diffusivity $D$ and solubility $S, K=D S$. Increasing $T$ at a fixed pressure drop $\Delta P$ causes the molecules to diffuse much faster, resulting in higher diffusivities. At high temperatures, there is also little, if any, possibility of adsorption of the gases on the pores' surface and, thus, the solubility decreases. Indeed, all of the density profiles presented in Figs. 5 and 6 verify that the amount 
of gas adsorbed in the membrane decreases with increasing $T$. Depending on whether the rate of increase of $D$ with $T$ is larger or smaller than the rate of the decreases in $S$, the overall fluxes may increases or decrease with $T$. Figure 8 presents the change in the fluxes of the gases with increasing temperature in the two binary mixtures, when an external pressure drop of $\Delta P=20$ has been applied to the membrane. As Fig. 8 indicates, in the present case the increase in $D$ is smaller than the decrease in $S$, so that the overall permeation rates (fluxes) decrease with $T$. Note, however, that since the membrane is subject to a large pressure gradient, $D$ is an effective diffusivity that represents the combined effect of pure diffusion and the convective effect.

Examination of the MD simulations, and in particular the pressure build-up in the two CVs, indicates that much fewer number of molecules are required at higher temperatures to build up the same pressure and, thus, the net fluxes are smaller. Note that fewer number of molecules implies a less "crowded" space, and thus higher $D$. It also implies smaller solubility.

Hydrogen is the lightest gas in the two binary mixtures and, therefore, we expect higher temperatures to result in higher separation factors for $\mathrm{H}_{2}$ over both $\mathrm{CO}_{2}$ and $\mathrm{CH}_{4}$. Figure 8 presents the results of the MD computations, which confirm the trends and indicate that the separation factor is always a increasing function of temperature.

\subsection{External pressure drop}

Figure 10 present the dependence on the external pressure drop $\Delta P$ of the separation factor for a membrane with the thicknesses $\ell=64.2 \AA$ for the two mixtures that we studied. For a membrane with a given thickness, the separation factor is not sensitive to the applied pressure drop, which justifies imposing in the simulations $\Delta P$ s that are larger than those used in the experiments.

\subsection{Membrane's thickness}

The separation factor of a membrane depends on its thickness $\ell$, but there is an optimal thickness $\ell_{o}$ such that, a thickness $\ell<\ell_{o}$ yields a separation factor smaller than the maximum separation that the membrane can attain, whereas the separation factor will not improve and may even decline again, if the membrane is thicker than its optimal thickness. Thus, we studied the dependence of the separation factor on the membrane's thickness by increasing it from $64.2 \AA$ to $385.2 \AA$, the largest thickness for which the SS condition could be reached using 
an affordable computation time. Figure 11 presents the results for the two binary mixtures when the applied pressure drop was $\Delta P=20$ atm, while Figures 12 and 13 show the same for $\Delta P=30$ and $40 \mathrm{~atm}$, respectively. Similar results were obtained for $\Delta P=50$ and 60 atm and are not shown. In all the cases, the separation factor increases with increasing the membrane's thickness. We then fitted the results to an exponential function,

$$
S(\ell)=S_{\infty}+A \exp (-\ell / B)
$$

where $A$ and $B$ are constants to be determined, and $S_{\infty}$ is the asymptotic separation factor, if we could increase the membrane's thickness to its experimental value. The fitting was carried out for each $\Delta P$. We find that values of $A$ are largely insensitive to the applied pressure drop.

The functional form of Eq. (9) deserves some discussions. Although Eq. (9) is, at this point, purely empirical and intended only for obtaining estimates of the separation factor as a function of the membrane's thickness (see the next section), it is not, in fact, without merit. As described earlier, since the model membrane is exposed to a large pressure gradient, convection plays an important role in the permeation process. If we solve the convection-diffusion equation for a porous medium of finite length $\ell$, we find that the concentration profile, and hence the effective diffusivity and the permeability, depend exponentially on the porous medium's length (see Eq. (11.76) of Ref. [10]). As a result, the separation factor, which is simply the ratio of the permeabilities, also depends exponentially on the porous medium length, or the membrane's thickness.

\subsection{Comparison with the experimental data}

As pointed out earlier, it is currently not feasible to carry out MD simulation of transport and separation of gaseous mixtures in an atomistic model of the $\mathrm{SiC}$ membranes with a thickness comparable with that of one that the USC group has been fabricating, which is in the range $[7,8] 2 \mu \mathrm{m}-6 \mu \mathrm{m}$. The experimental separation factors are [7,8] 93 and 104 for the $\mathrm{H}_{2} / \mathrm{CO}_{2}$ $\mathrm{H}_{2} / \mathrm{CH}_{4}$ mixtures, respectively, measured at $T=475 \mathrm{~K}$. If the trends indicated by Eq. (7) are correct, then, setting $S_{\infty}$ to its experimental value should yield a membrane thickness comparable with that of the fabricated membranes. In Table 2 we list the estimated membrane's thickness, using the experimental $S_{\infty}$, for the two mixtures for three external pressure drops $\Delta P$. The estimated thicknesses fall very nicely within the range of experimental thickness of 
the membranes. Ultimately, of course, one must increase the thickness of the atomistic model of the membrane to much higher values, in order to obtain more accurate estimates of the parameters $A$ and $B$, and possibly $S_{\infty}$. But, the model developed in Parts I and II and utilized here for the simulation of transport and separation of the two binary gaseous mixtures provide all the correct experimental trends, as well as an accurate estimate the optimal thickness of the membrane, which are very encouraging.

\section{Summary}

We utilized the atomistic model of amorphous silicon-carbide membrane, developed in Parts I and II of this series, to study transport and separation of equimolar $\mathrm{H}_{2} / \mathrm{CO}_{2}$ and $\mathrm{H}_{2} / \mathrm{CH}_{4}$ gaseous mixtures at high temperatures. The dual control volume-grand canonical molecular dynamics simulation was used in order to simulate the transport and separation processes. Membranes of up to $38 \mathrm{~nm}$ in thickness were simulated, and the effect of the various parameters on their separation properties is investigated. They include the temperature, the applied pressure drop across the system, and the membrane thickness. The separation factors are insensitive to the pressure drop applied across the membrane, while they are strong functions of the temperature and the membrane's thickness. The trends in the dependence of the separation factor on the membrane's thickness are consistent with what is obtained in experiments. If the dependence of the computed separation factors on the membranes thickness and the experimental values of the separation factors are used as input, the simulations provide estimates of the thickness of the membranes that are in agreement with the experimental data, hence providing more evidence of the accuracy of the atomistic model.

\section{Acknowledgments}

Work at USC was supported by the Office of Energy Sciences, Chemical Sciences, Geosciences \& Biosciences Division of the Department of Energy. Work at Caltech was supported by the Department of Transportation, grant DTFH61-10-C00019. 


\section{References}

1. S. Naserifar, L. Liu, W. A. Goddard, III, T. T. Tsotsis, M. Sahimi, Toward a processbased molecular model of $\mathrm{SiC}$ membranes. I. Development of a reactive force field, J. Phys. Chem. C 117 (2013) 3308.

2. A. C. T. van Duin, S. Dasgupta, F. Lorant, W. A. Goddard, III, ReaxFF: A reactive force field for hydrocarbons, J. Phys. Chem. A 105 (2001) 9396.

3. A. Jaramillo-Botero, S. Naserifar, W. A. Goddard, III, A general multi-objective force field optimization framework and its application to the design of reactive force fields for silicon carbide, J. Chem. Theory Comput. 10 (2014) 1426.

4. S. Naserifar, W. A. Goddard, III, L. Liu, T. T. Tsotsis, M. Sahimi, Toward a process-based molecular model of $\mathrm{SiC}$ membranes. II. Reactive dynamics simulation of the pyrolysis of polymer precursor to form amorphous SiC, J. Phys. Chem. C 117 (2013) 3320.

5. V. Suwanmethanond, E. Goo, P. K. T. Liu, G. Johnston, M. Sahimi, T. T. Tsotsis, Porous silicon carbide sintered substrates for high-temperature membranes, Ind. Eng. Chem. Res. 39 (2000) 3264.

6. R. Ciora, B. Fayyaz, P. K. T. Liu, V. Suwanmethanond, R. Mallada, M. Sahimi, T. T. Tsotsis, Preparation and reactive applications of nanoporous silicon carbide membranes, Chem. Eng. Sci. (2004) 4957.

7. B. Elyassi, M. Sahimi, T. T. Tsotsis, Silicon carbide membranes for gas separation applications, J. Membr. Sci. 288 (2007) 290.

8. B. Elyassi, M. Sahimi, T. T. Tsotsis, A novel sacrificial interlayer-based method for the preparation of silicon carbide membranes, J. Membr. Sci. 316 (2008) 73.

9. B. Elyassi, W. Deng, M. Sahimi, T. T. Tsotsis, On the use of porous and nonporous fillers in the fabrication of silicon carbide membranes, Ind. Eng. Chem. Res. 52 (2013) 10269.

10. M. Sahimi, Flow and Transport in Porous Media and Fractured Rock, 2nd ed. (WileyVCH, Weinheim, 2011). 
11. M. Sahimi, in Advances in Membrane Characterization, J. Pellegrino, ed. (Wiley, New York, in press, 2015).

12. G. S. Heffelfinger, F. van Swol, Diffusion in Lennard-Jones fluids using dual control volume grand canonical molecular dynamics simulation DCV-GCMD, J. Chem. Phys. 100 (1994) 7548 .

13. J. M. D. MacElroy, Nonequilibrium molecular dynamics simulation of diffusion and flow in thin microporous membranes, J. Chem. Phys. 101 (1994) 5274.

14. R. F. Cracknell, D. Nicholson, N. Quirke, Direct molecular dynamics simulation of flow down a chemical potential gradient in a slit-shaped micropore, Phys. Rev. Lett 74 (1995) 2463.

15. L. Xu, M. G. Sedigh, M. Sahimi, T. T. Tsotsis, Nonequilibrium molecular dynamics simulation of transport of gas mixtures in nanopores, Phys. Rev. Lett. 80 (1998) 3511.

16. L. Xu, M. G. Sedigh, T. T. Tsotsis, M. Sahimi, Nonequilibrium molecular dynamics simulation of transport and separation of gases in carbon nanopores. II. Binary and ternary mixtures and comparison with the experimental data, J. Chem. Phys. 112 (2000) 910 .

17. L. Xu, M. Sahimi, T. T. Tsotsis, Nonequilibrium molecular dynamics simulations of transport and separation of gas mixtures in nanoporous materials, Phys. Rev. E 62 (2000) 6942.

18. M. Firouzi, T. T. Tsotsis, M. Sahimi, Nonequilibrium molecular dynamics simulations of transport and separation of supercritical fluid mixtures in nanoporous membranes. I. Results for a single carbon nanopore, J. Chem. Phys. 119 (2003) 6810.

19. M. Firouzi, M. Sahimi, T. T. Tsotsis, Supercritical fluids in porous composite materials: Direction-dependent flow properties, Phys. Rev. E 73 (2006) 036312/1.

20. N. Rajabbeigi, T. T. Tsotsis, M. Sahimi, Molecular pore-network model for nanoporous materials. II: Application to transport and separation of gaseous mixtures in siliconcarbide membranes, J. Membr. Sci. 345 (2009) 323. 
21. G. Soave, Equilibrium constants from a modified Redlich-Kwong equation of state, Chem. Eng. Sci. 27 (1972) 1197.

22. A. Spitzbart, A generalization of Hermite's interpolation formula, Am. Math. Monthly 67 (1960) 42.

23. S. Kirkpatrick, C. D. Gelatt, M. P. Vecchi, Optimization by simulated annealing, Science $220(1983) 671$.

24. S. Naserifar, W. A. Goddard, III, M. Sahimi, T. T. Tsotsis, Phys. Rev. E, to be published. 
Table 1: The Lennard-Jones parameters for the various gases and membrane atoms, used in the DCV-GCMD simulations.

\begin{tabular}{llll}
\hline Gases and atoms & $\sigma(\AA)$ & $\epsilon / k_{B}(\mathrm{~K})$ & Mass \\
\hline $\mathrm{C}$ & 3.400 & 28.0 & 12.00 \\
$\mathrm{Si}$ & 3.742 & 23.6 & 28.09 \\
$\mathrm{H}_{2}$ & 2.827 & 59.7 & 2.04 \\
$\mathrm{CO}_{2}$ & 3.794 & 225.3 & 44.01 \\
$\mathrm{CH}_{4}$ & 3.810 & 148.1 & 16.04 \\
\hline
\end{tabular}

Table 2: Predicted values of the membrane thickness for the $\mathrm{H}_{2} / \mathrm{CO}_{2}$ and $\mathrm{H}_{2} / \mathrm{CH}_{4}$ mixtures for the pressure drops $\Delta P=20$ and 60 atm. The temperature is $200^{\circ} \mathrm{C}$.

\begin{tabular}{lcc}
\hline mixture & $\Delta P($ atm $)$ & optimal thickness $(\mu \mathrm{m})$ \\
\hline $\mathrm{H}_{2} / \mathrm{CO}_{2}$ & 20 & 1.79 \\
$\mathrm{H}_{2} / \mathrm{CO}_{2}$ & 60 & 3.83 \\
$\mathrm{H}_{2} / \mathrm{CH}_{4}$ & 20 & 4.03 \\
$\mathrm{H}_{2} / \mathrm{CH}_{4}$ & 60 & 6.64 \\
\hline
\end{tabular}

\section{Captions}

Figure 1. Schematic illustration of the simulation system with the $\mathrm{SiC}$ membrane in the middle. The $h$ and $l$ areas represent, respectively, the high- and low-pressure control volumes.

Figure 2. Time-dependance of the separation factor of the membrane for the $\mathrm{H}_{2} / \mathrm{CO}_{2}$ mixture, exposed to the various pressures drops.

Figure 3. Same as in Figure 2, but for the $\mathrm{H}_{2} / \mathrm{CH}_{4}$ mixture.

Figure 4. The dimensionless density profiles of the $\mathrm{H}_{2} / \mathrm{CO}_{2}$ mixture in the axial $\left(X^{*}\right)$ direction in the membrane (middle region) and the two control volumes. The profiles represent averages over the cross section of the membrane.

Figure 5. Same as in Figure 4, but for higher pressure drops.

Figure 6. The average dimensionless temperature $T^{*}$ in the membrane (middle) and the two control volumes. Numbers in the parentheses indicate the set temperatures. The applied external pressure drop is 20 atm. 
Figure 7. The dimensionless density profiles of the $\mathrm{H}_{2} / \mathrm{CH}_{4}$ mixture in the axial $(X)$ direction. The profiles represent averages over the cross section of the membrane.

Figure 8. Temperature-dependence of the separation factor for the two gaseous mixtures.

Figure 9. Temperature-dependence of the fluxes of the two gaseous mixtures.

Figure 10. Effect of the applied external pressure drop on the separation factor for a membrane of thickness $64.2 \AA$.

Figure 11. Effect of the membrane's thickness $\ell$ on its separation factor for the two gaseous mixtures. The lines represent the fit of the data to Eq. (9), with $A \simeq 91.72$ and $B \simeq 0.39$ for the $\mathrm{H}_{2} / \mathrm{CO}_{2}$ mixture, and $A \simeq 101.93$ and $B \simeq 0.84$ for the $\mathrm{H}_{2} / \mathrm{CH}_{4}$ mixture, both at at $T=475 \mathrm{~K}$.

Figure 12. Same as in Figure 11, but for an external pressure drop of $30 \mathrm{~atm}$. The fitting parameters are $A \simeq 91.95$ and $B \simeq 0.42$ for the $\mathrm{H}_{2} / \mathrm{CO}_{2}$ mixture, and $A \simeq 101.74$ and $B \simeq 1.06$ for the $\mathrm{H}_{2} / \mathrm{CH}_{4}$ mixture.

Figure 13. Same as in Figure 11, but for an external pressure drop of $40 \mathrm{~atm}$. The fitting parameters are $A \simeq 91.86$ and $B \simeq 0.53$ for the $\mathrm{H}_{2} / \mathrm{CO}_{2}$ mixture, and $A \simeq 101.44$ and $B \simeq 2.11$ for the $\mathrm{H}_{2} / \mathrm{CH}_{4}$ mixture. 


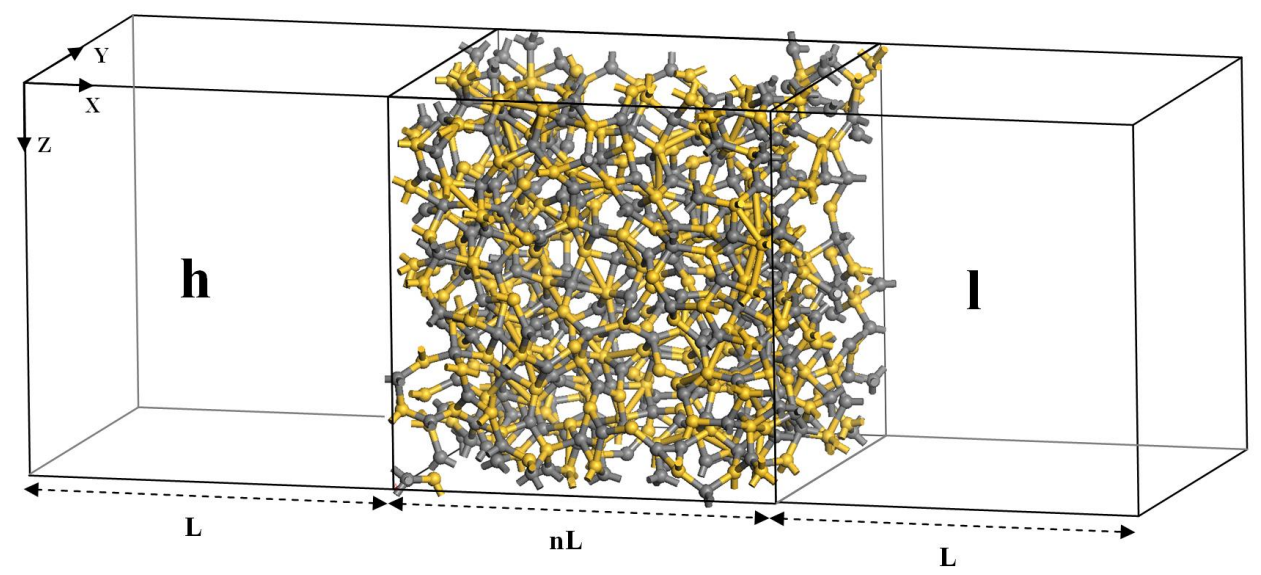

Figure 1: Schematic illustration of the simulation system with the $\mathrm{SiC}$ membrane in the middle. The $h$ and $l$ areas represent, respectively, the high- and low-pressure control volumes.

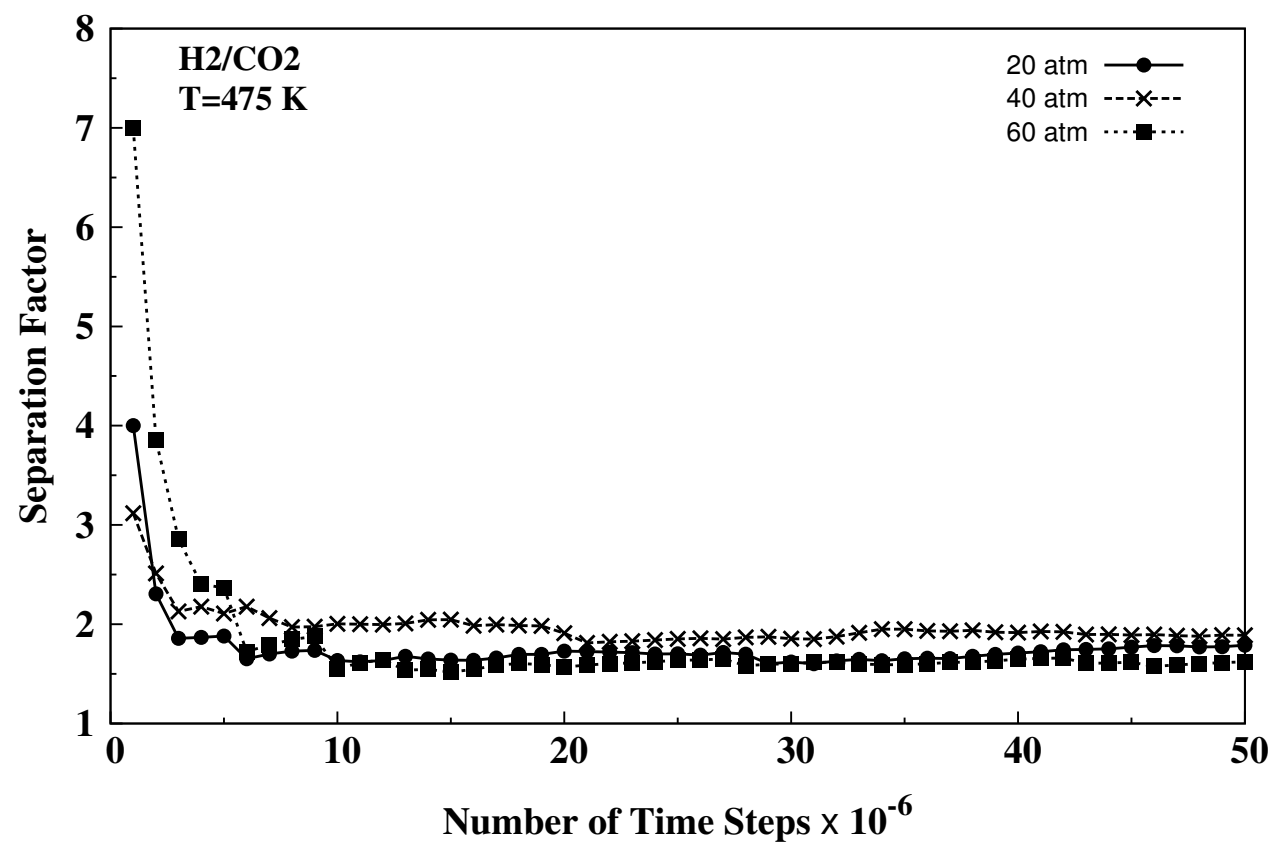

Figure 2: Time-dependance of the separation factor of the membrane for the $\mathrm{H}_{2} / \mathrm{CO}_{2}$ mixture, exposed to the various pressures drops. 


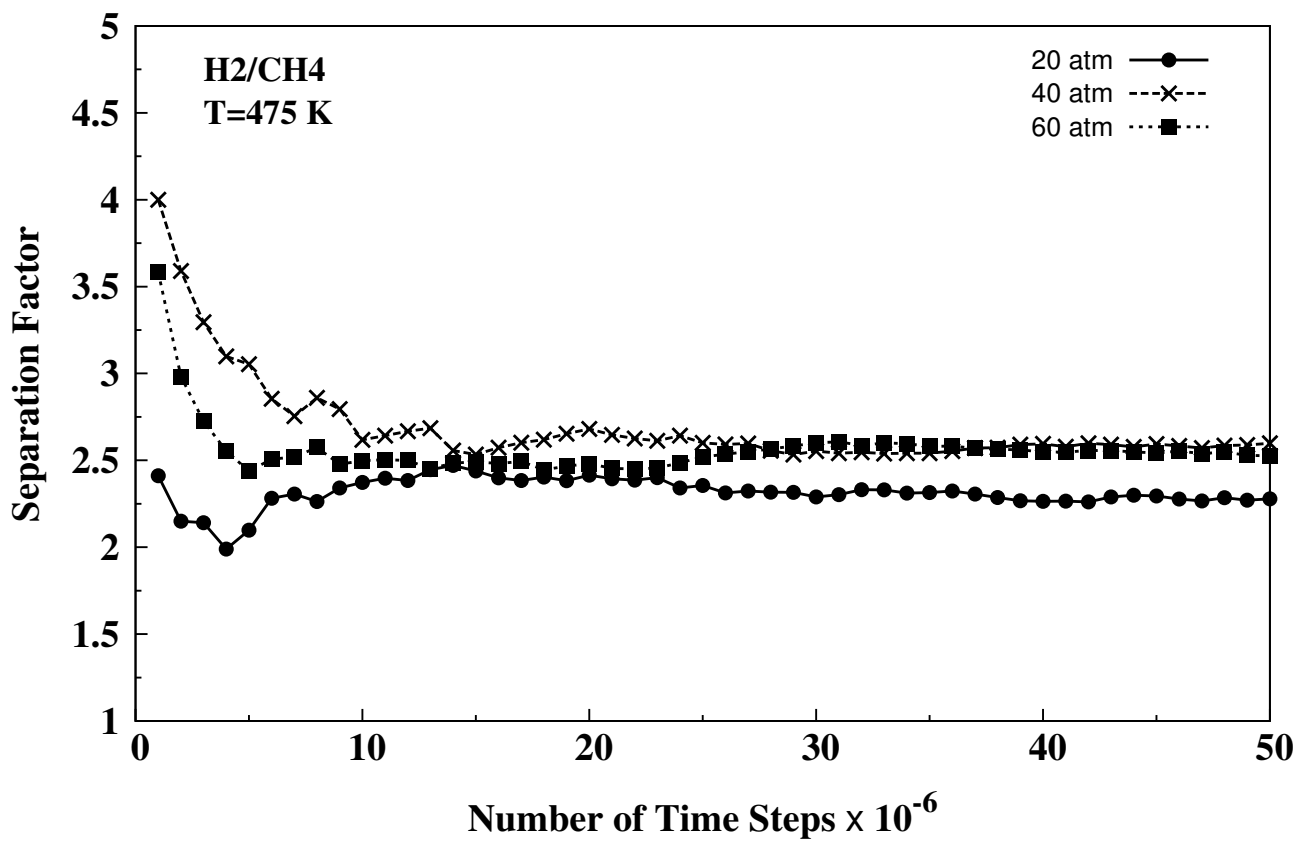

Figure 3: Same as in Figure 2, but for the $\mathrm{H}_{2} / \mathrm{CH}_{4}$ mixture. 
Figure 4: The dimensionless density profiles of the $\mathrm{H}_{2} / \mathrm{CO}_{2}$ mixture in the axial $(X)$ direction in the membrane (middle region) and the two control volumes. The profiles represent averages over the cross section of the membrane.
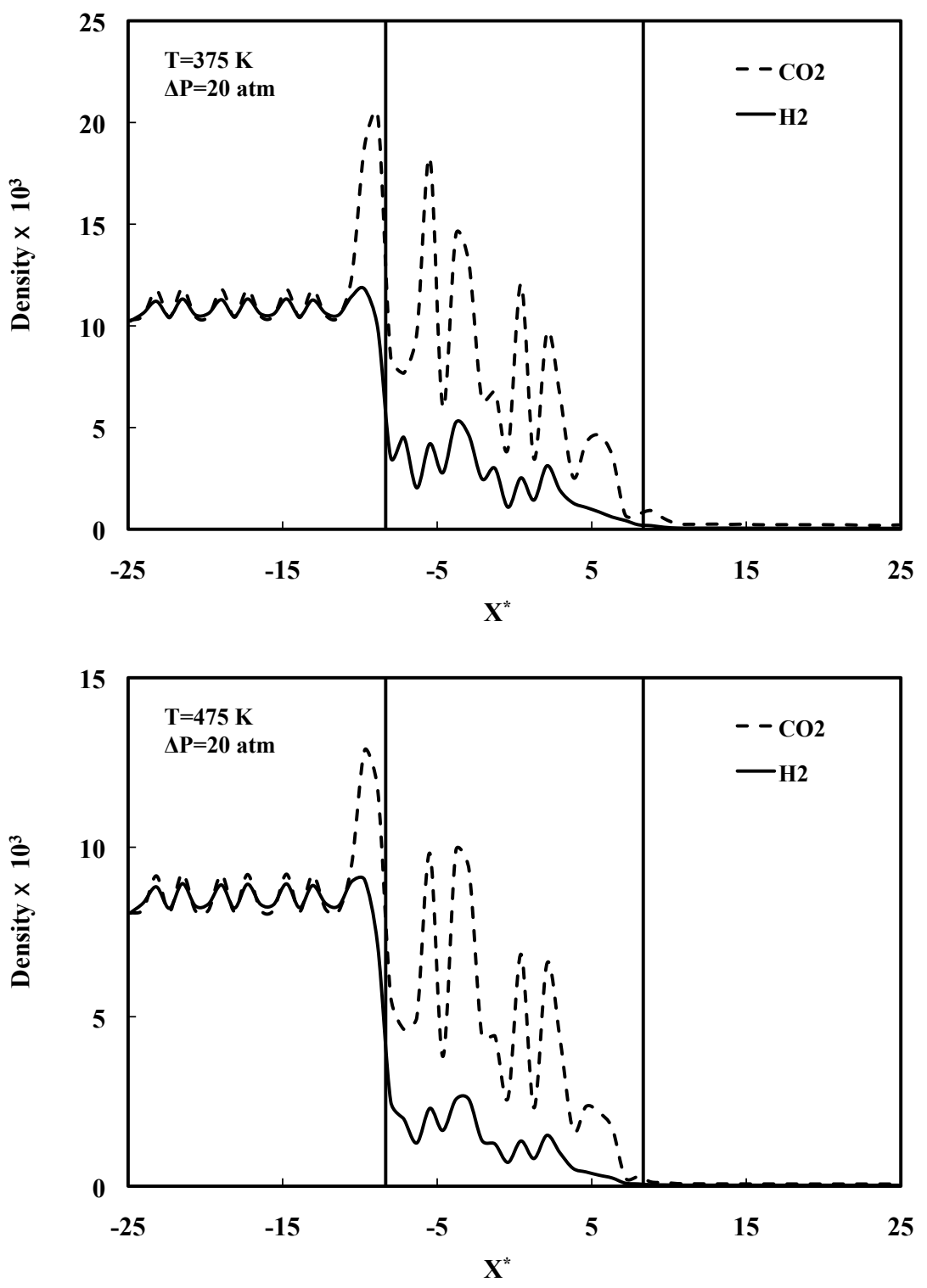

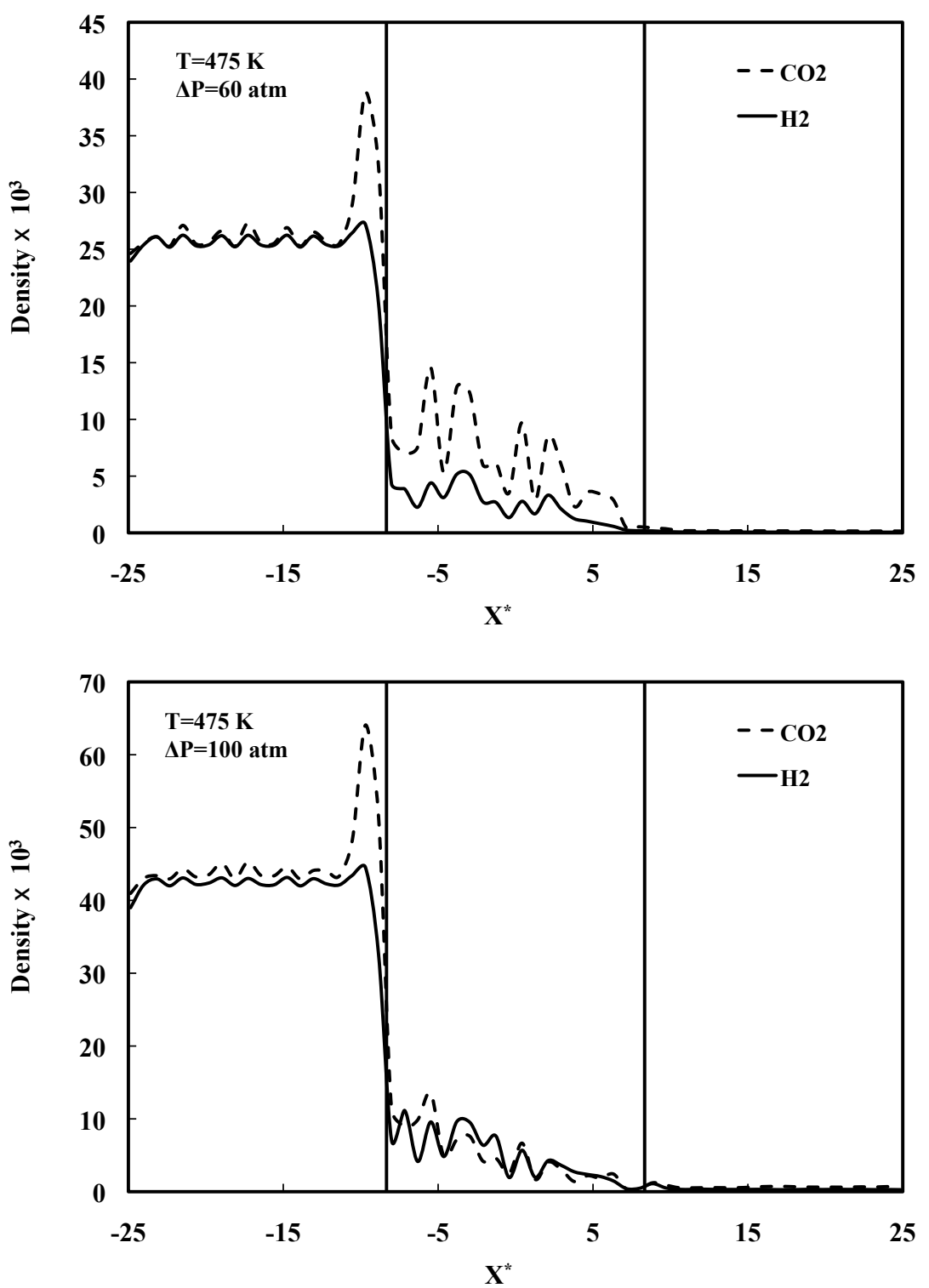

Figure 5: Same as in Figure 4, but for higher pressure drops. 

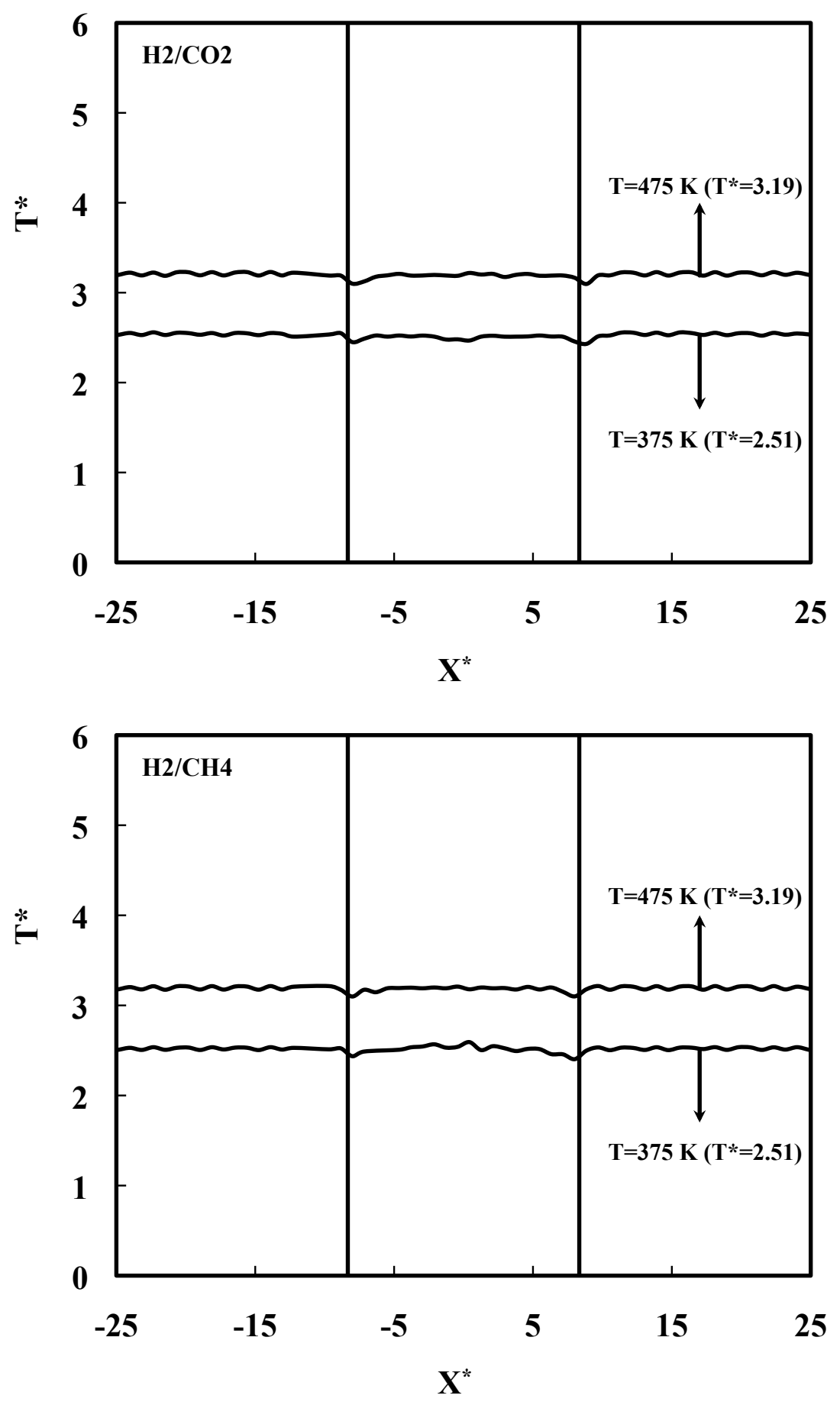

Figure 6: The average dimensionless temperature $T^{*}$ in the membrane (middle) and the two control volumes. Numbers in the parentheses indicate the set temperatures. The applied external pressure drop is 20 atm. 

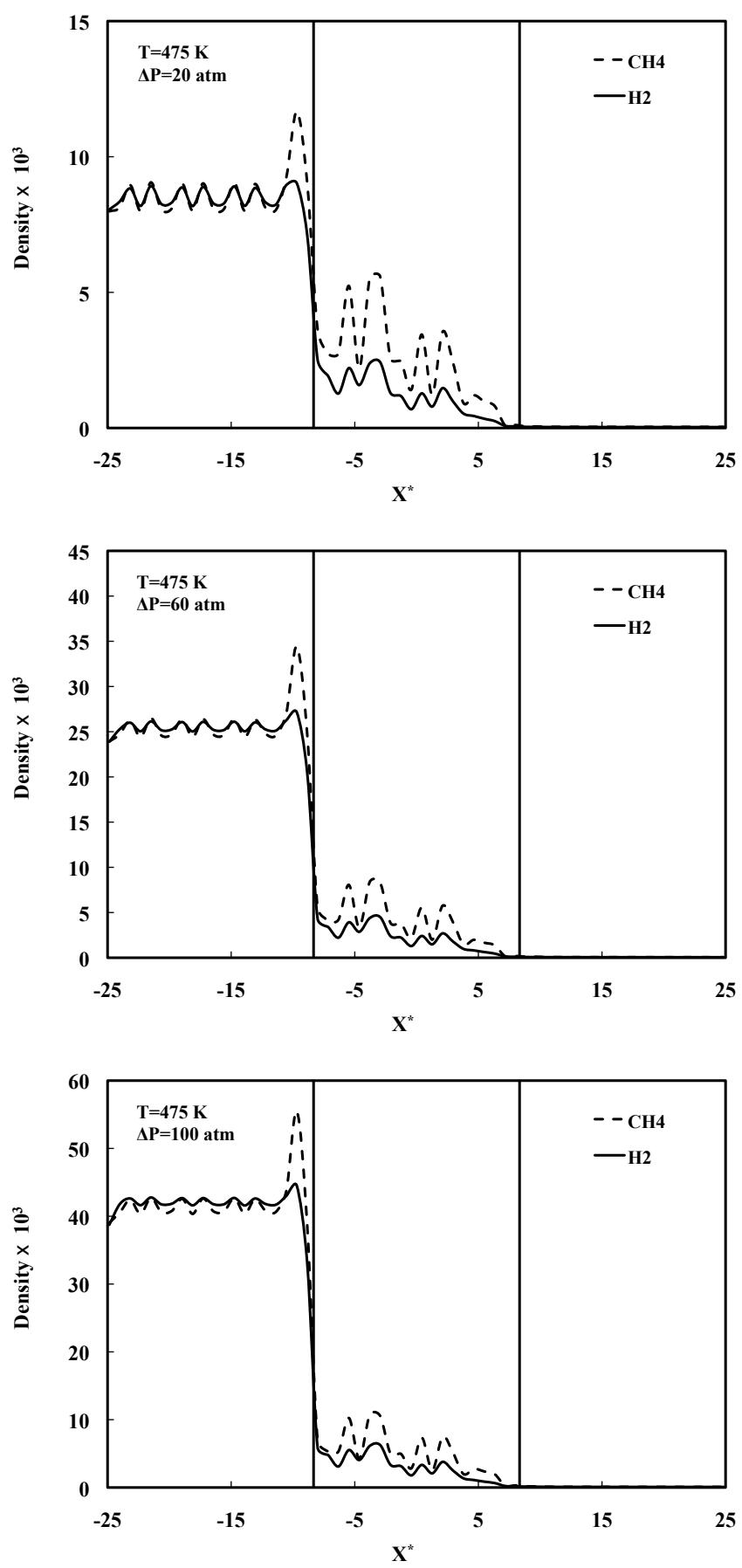

Figure 7: The dimensionless density profiles of the $\mathrm{H}_{2} / \mathrm{CH}_{4}$ mixture in the axial $(X)$ direction. The profiles represent averages over the cross section of the membrane. 


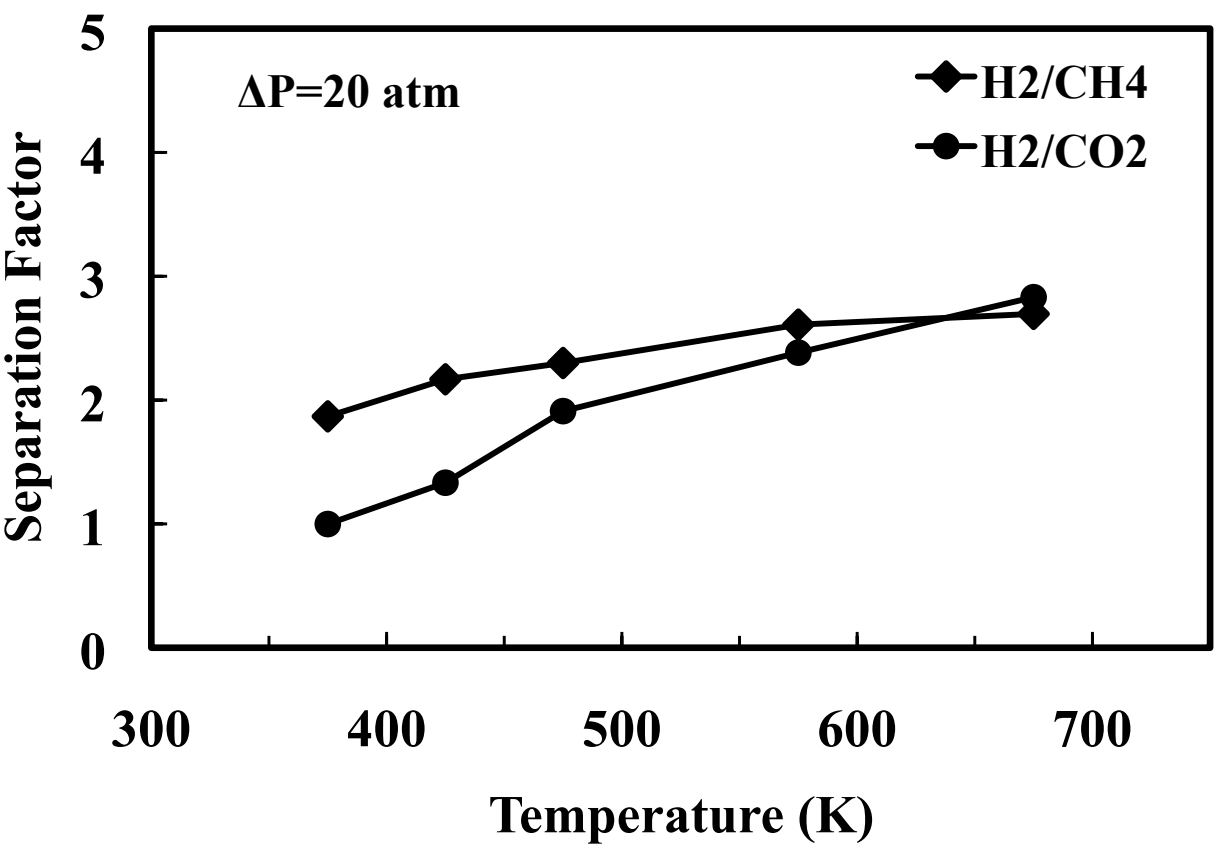

Figure 8: Temperature-dependence of the separation factor for the two gaseous mixtures. 

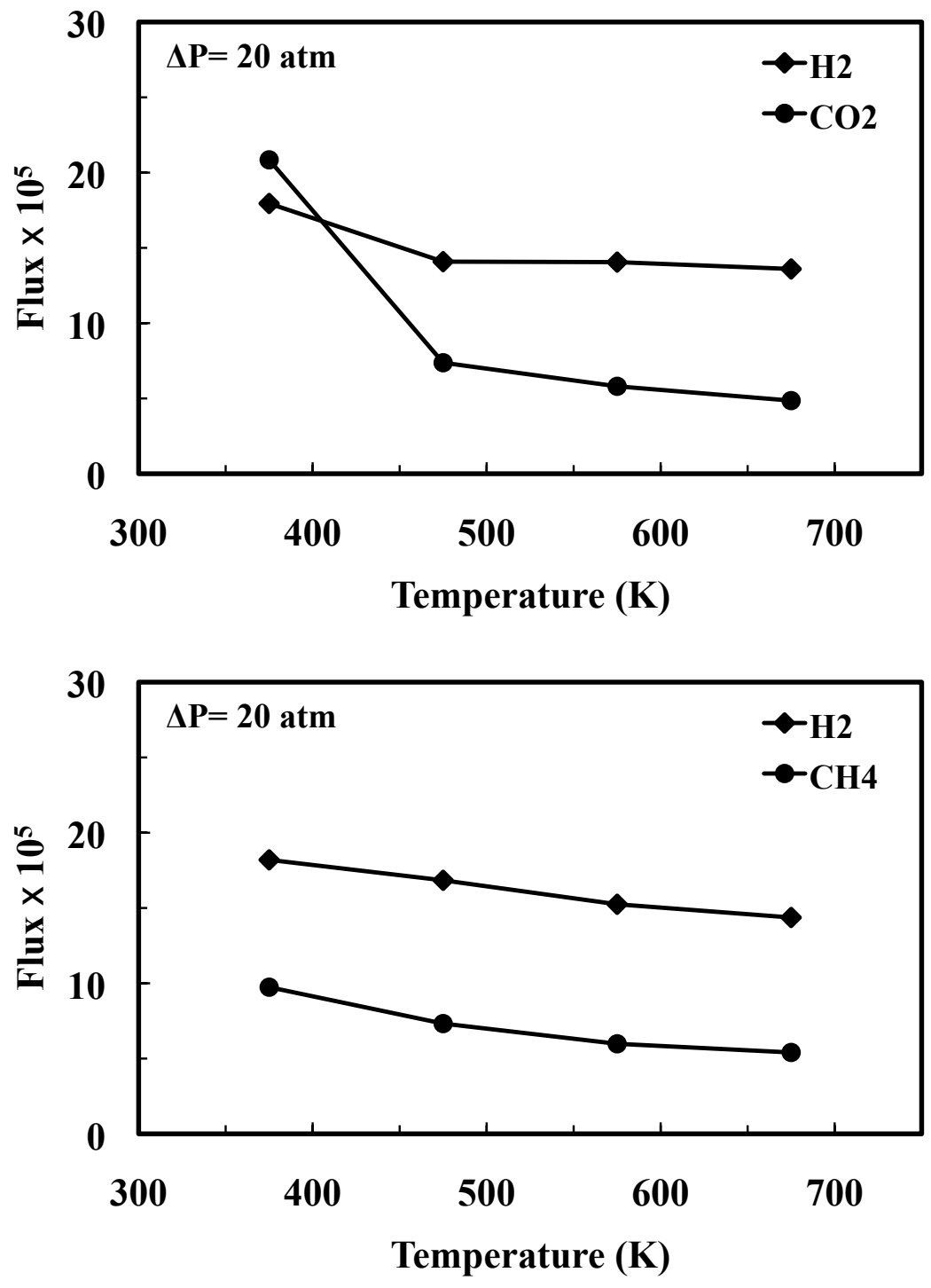

Figure 9: Temperature-dependence of the fluxes of the two gaseous mixtures. 


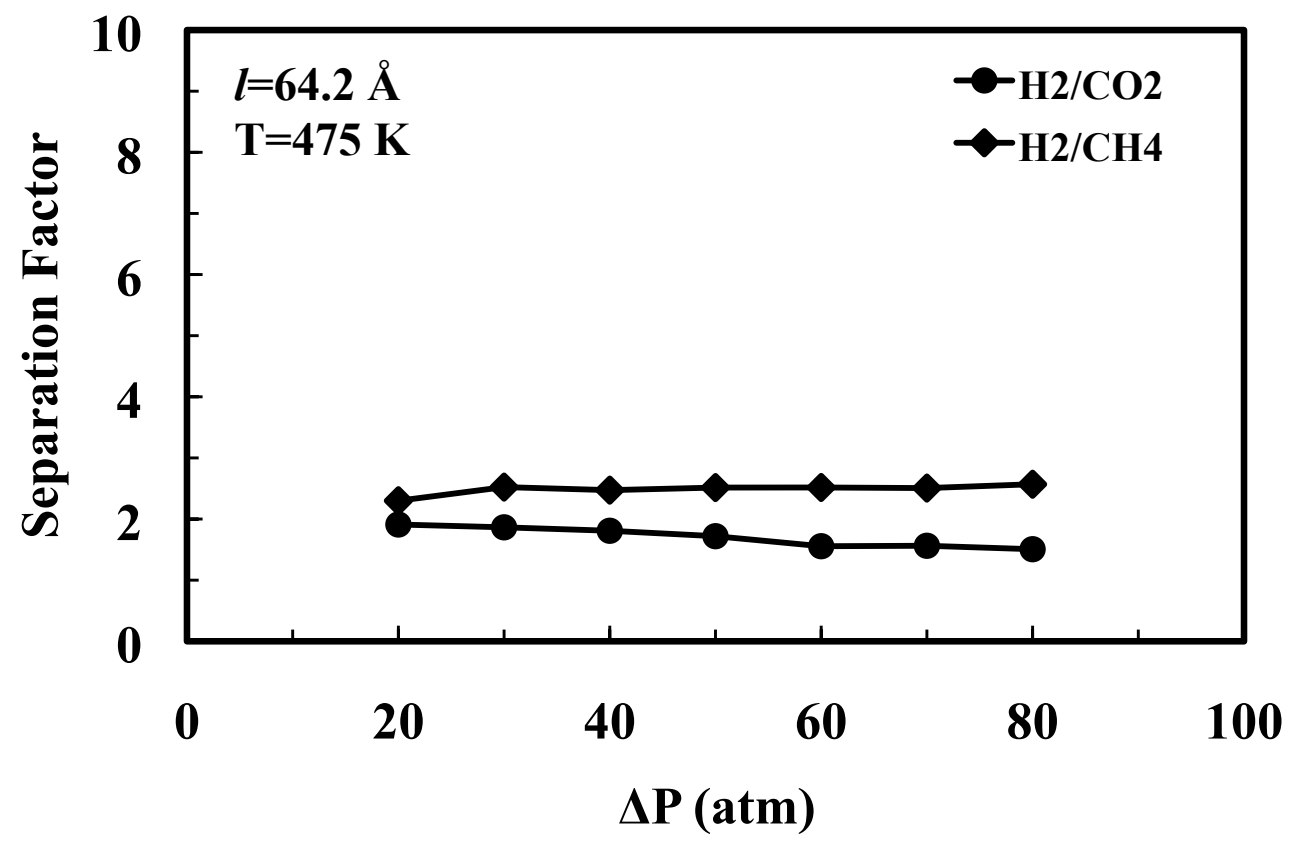

Figure 10: Effect of the applied external pressure drop on the separation factor for a membrane of thickness $64.2 \AA$. 

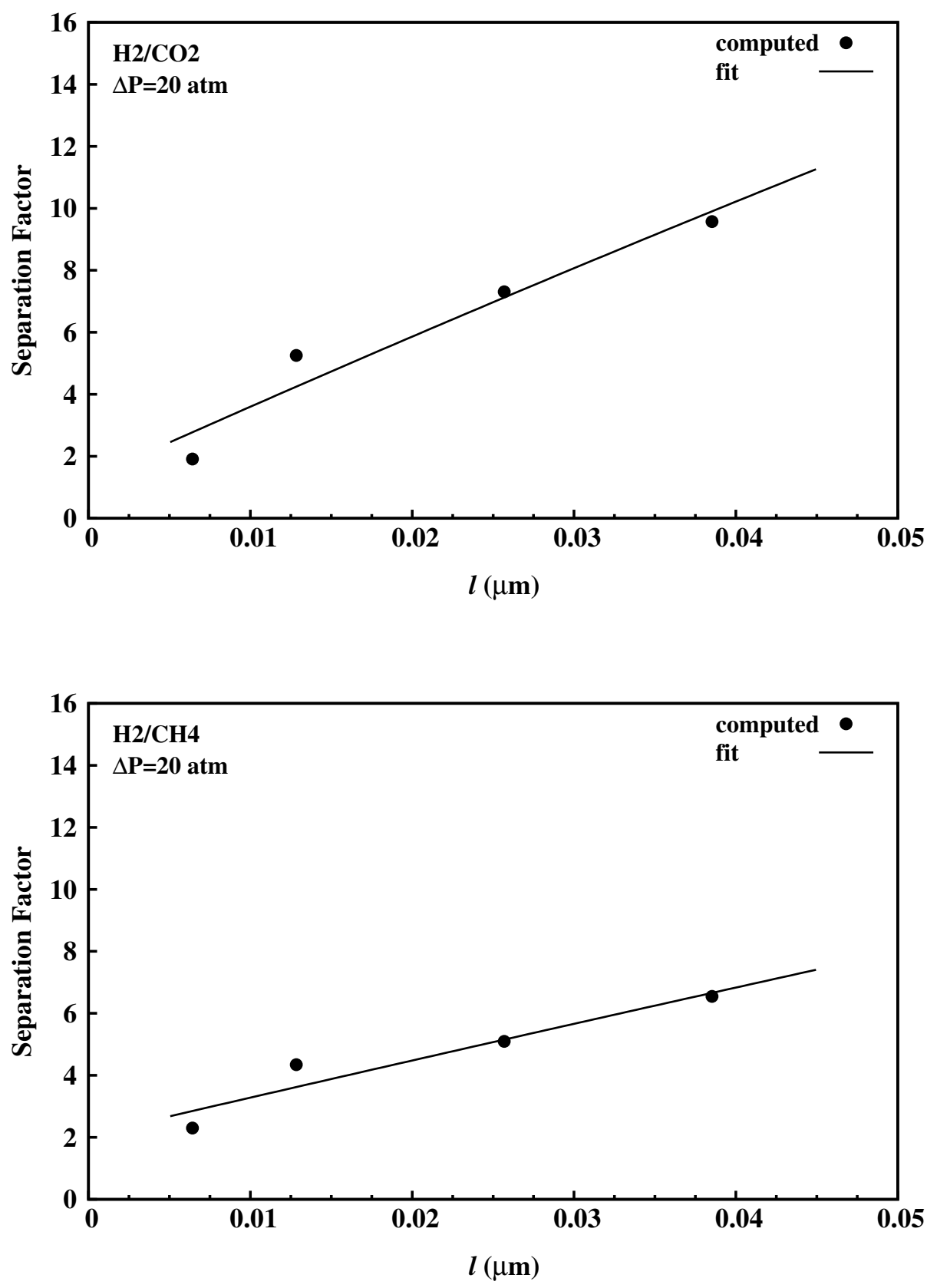

Figure 11: Effect of the membrane's thickness $\ell$ on its separation factor for the two gaseous mixtures. The lines represent the fit of the data to Eq. (9), with $A \simeq 91.72$ and $B \simeq 0.39$ for the $\mathrm{H}_{2} / \mathrm{CO}_{2}$ mixture, and $A \simeq 101.93$ and $B \simeq 0.84$ for the $\mathrm{H}_{2} / \mathrm{CH}_{4}$ mixture, both at $T=475$ $\mathrm{K}$. 


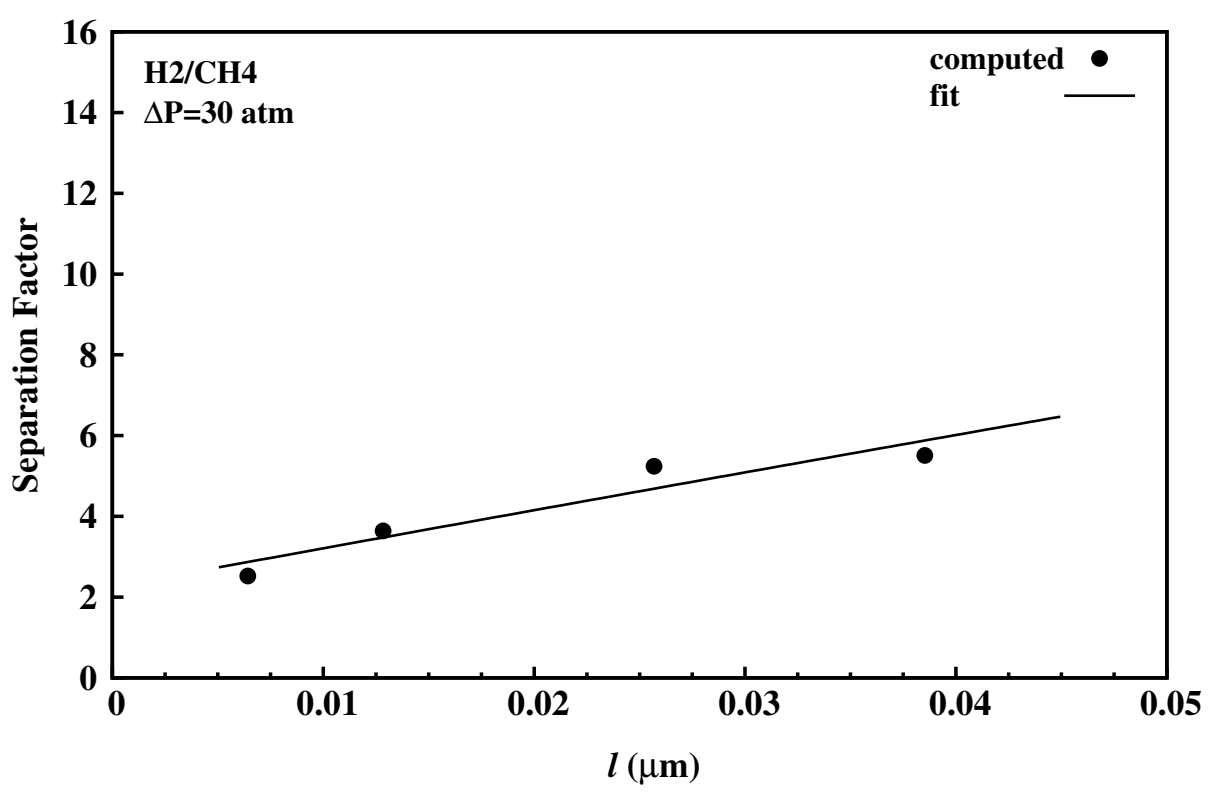

Figure 12: Same as in Figure 11, but for an external pressure drop of $30 \mathrm{~atm}$. The fitting parameters are $A \simeq 91.95$ and $B \simeq 0.42$ for the $\mathrm{H}_{2} / \mathrm{CO}_{2}$ mixture, and $A \simeq 101.74$ and $B \simeq 1.06$ for the $\mathrm{H}_{2} / \mathrm{CH}_{4}$ mixture. 


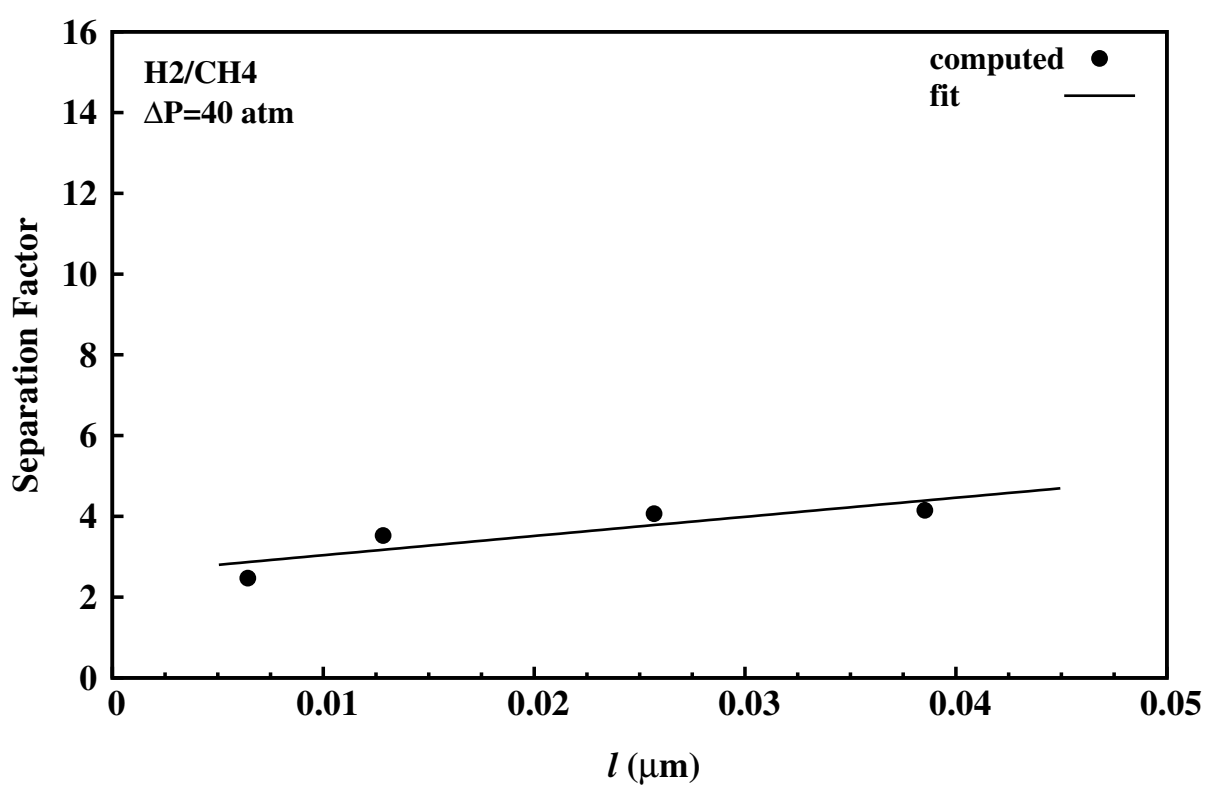

Figure 13: Same as in Figure 11, but for an external pressure drop of $40 \mathrm{~atm}$. The fitting parameters are $A \simeq 91.86$ and $B \simeq 0.53$ for the $\mathrm{H}_{2} / \mathrm{CO}_{2}$ mixture, and $A \simeq 101.44$ and $B \simeq 2.11$ for the $\mathrm{H}_{2} / \mathrm{CH}_{4}$ mixture. 


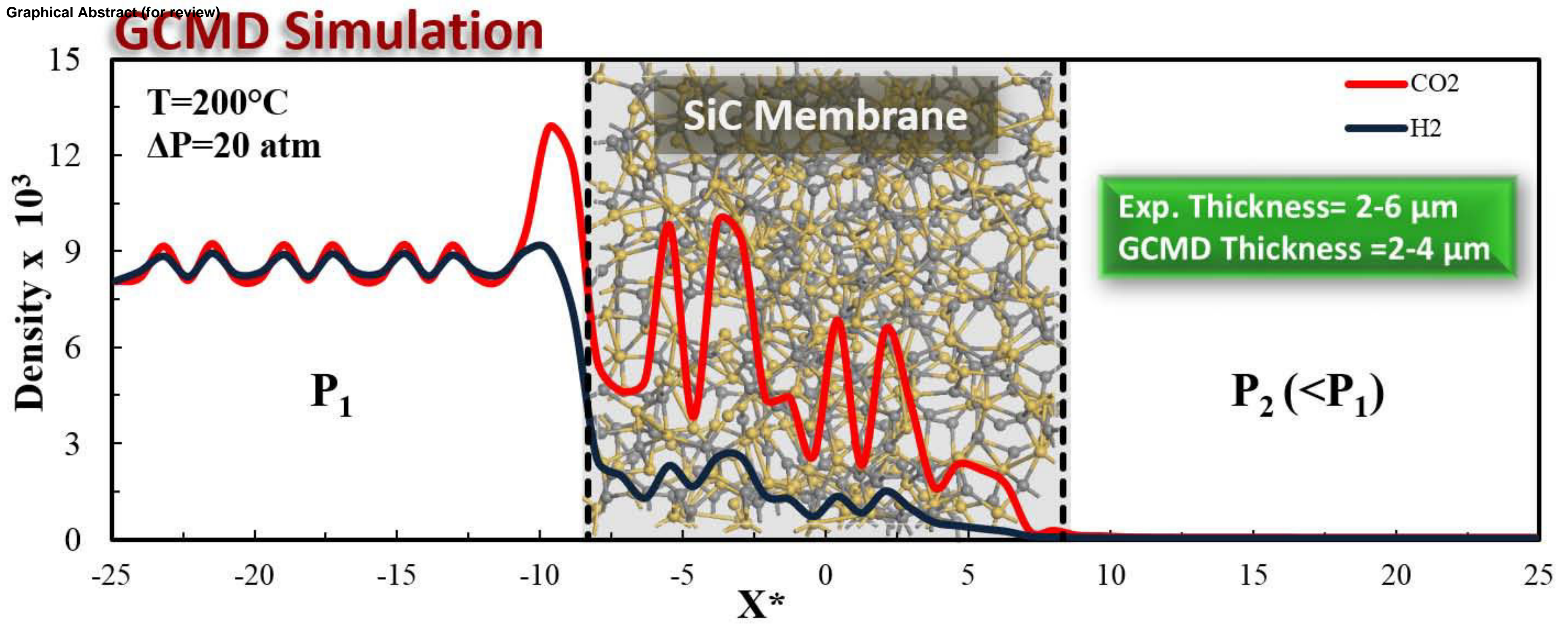

